\title{
Dados Psicométricos Adicionais da Versão Portuguesa do Questionário de Experiências Depressivas
}

\author{
Additional Psychometric Data with the Portuguese Version of the Depressive \\ Experiences Questionnaire
}

\author{
Rui C. Campos ${ }^{1}$
}

\begin{abstract}
Resumo
O Questionário de Experiências Depressivas (QED, Blatt, D’Afflitti, \& Quinlan, 1976) é uma medida de auto-relato usada na avaliação e na investigação sobre a personalidade e a psicopatologia. Avalia os constructos de Dependência, Auto-criticismo, Necessidade, Contacto e Eficácia. A versão portuguesa do QED foi previamente estabelecida por Campos, Besser e Blatt (2013). O presente estudo expande o estudo anterior, apresentando dados psicométricos para a versão portuguesa do QED com duas amostras: uma amostra extensa da comunidade, de 1545 adultos, e uma amostra de 165 jovens adultos, permitindo testar a consistência interna, a validade convergente e a estabilidade temporal da versão portuguesa do QED. Os resultados obtidos complementam os resultados obtidos previamente e confirmam a precisão e a validade da versão portuguesa do QED.
\end{abstract}

Palavras-chave: Questionário de Experiências Depressivas, adaptação portuguesa, auto-criticismo, necessidade, validade

\begin{abstract}
The Depressive Experiences Questionnaire (QED, Blatt, D'Afflitti, \& Quinlan, 1976) is a self-report measure used in personality and psychopathology assessment and research. It measures the constructs of Dependency, Self-criticism, Neediness, Connectedness and Efficacy. The Portuguese version of the DEQ has been previously established by Campos, Besser, and Blatt (2013). The present study expands the previous study presenting additional psychometric data for the Portuguese version of the DEQ with two samples: a large community sample of 1545 adults and a sample of 165 young adults, allowing testing for internal consistency, convergent validity and test-retest stability. Findings complement previous results and confirm the reliability and validity of the Portuguese version of the DEQ.
\end{abstract}

Keywords: Depressive Experiences Questionnaire, portuguese adaptation, self-criticism, neediness, validity

Agradecimentos: Gostaríamos de agradecer aos assistentes de investigação pelo seu importante trabalho de recolha de dados. Gostaríamos igualmente de agradecer a todos os participantes neste estudo.

${ }^{1}$ Departamento de Psicologia, Escola de Ciências Sociais e Centro de Investigação em Educação e Psicologia (CIEP), Universidade de Évora, Apartado 94, 7702.554 Évora, Portugal. Tel.: +351266768050. Fax: +351266768073. E-mail: rcampos@uevora.pt 


\section{Introdução}

O desenvolvimento da personalidade envolve uma integração de dois processos ou dimensões psicológicas: o relacionamento e a auto-definição (Blatt, 2008). Quando o processo normal e sinérgico de desenvolvimento é perturbado e uma ênfase excessiva é colocada num dos dois processos, ocorre uma expressão de níveis elevados de Dependência/Necessidade e/ou de Auto-criticismo (Blatt, 2004; Blatt \& Homann, 1992; Coyne \& Whiffen, 1995; Quimette \& Klein, 1993), conduzindo a um aumento da vulnerabilidade à depressão e ao mal-estar psicológico (distress) (Blatt \& Zuroff, 1992).

O Questionário de Experiências Depressivas (QED; Blatt, D’Afflitti, \& Quinlan, 1976) é um questionário composto por 66 itens que avaliam experiências quotidianas de indivíduos depressivos, mas não sintomas de depressão. $\mathrm{O}$ QED foi desenvolvido num quadro conceptual psicodinâmico que assume uma continuidade entre o afecto depressivo em populações clínicas e não clínicas (Blatt, 1974). Com base numa revisão da literatura clássica sobre a depressão (e.g., Bibring, 1953; Cohen, Baker, Cohen, FromReichman, \& Weigert, 1954; Freud, 1917/1980), Blatt e colegas reuniram 120 afirmações que se referiam a experiências quotidianas descritas por indivíduos depressivos. Sessenta e seis destas afirmações foram seleccionadas por diversos juízes, dado que descreviam experiências fenomenológicas típicas da depressão. Estas afirmações foram escritas sob a forma de itens de inventário para serem respondidos em escalas de Likert de 7 pontos, de discordo totalmente a concordo totalmente.

O QED proporciona resultados para dois factores: Dependência e Auto-criticismo (Blatt et al., 1976), que são consistentes com as duas dimensões depressivas previamente discutidas por Blatt (1974). Proporciona ainda um resultado para um terceiro factor: Eficácia. Estes três factores derivaram de uma Análise em Componentes Principais conduzida com uma amostra de jovens adultos americanos (Blatt et al., 1976). Esta estrutura factorial foi replicada com um grande número de amostras em diferentes culturas e línguas (Blatt, 2004).
O primeiro factor do QED, Dependência, inclui itens relativos às relações interpessoais, envolvendo temas como a preocupação com a possibilidade de ser abandonado ou rejeitado, perder outros significativos, a solidão e o desamparo, o querer estar perto e depender dos outros. Envolvem também preocupações com a possibilidade de ofender ou magoar alguém, o que conduz à dificuldade em expressar a agressividade por medo de perder a gratificação que os outros proporcionam (e. g., "Muitas vezes sinto-me desamparado(a)"). Os itens que saturam no segundo factor, Auto-criticismo, expressam sentimentos de culpa, vazio interior, desesperança, insatisfação, insegurança e o sentimento de não viver de acordo com as expectativas. Expressam igualmente dificuldades em assumir responsabilidade, o sentimento de ameaça perante a mudança, sentimentos de ambivalência em relação ao Self e aos outros, a tendência para assumir a culpa e para ser auto-crítico (e.g., "Sinto-me culpado com frequência"). Os itens do factor de Eficácia reflectem esforços dirigidos para objectivos, mas não uma competição excessiva com os outros (e.g., "Estou muito satisfeito comigo próprio(a) e com as minhas realizações pessoais").

A maioria dos estudos com o QED focou-se nos dois primeiros factores, dada a sua relevância para o funcionamento psicológico desadaptativo. Para obter as pontuações nos três factores, cada item do QED é multiplicado pelo seu coeficiente factorial em cada um dos factores. A pontuação total em cada factor do QED é obtida somando os valores ponderados dos 66 itens. Assim sendo, cada item entra na cotação dos três factores, tendo em conta o seu peso relativo em cada factor.

Diversos estudos demonstraram qualidades psicométricas aceitáveis do QED quer em amostras clínicas, quer em amostras não clínicas (veja-se Blatt, 2004; Desmet et al., 2007; Desmet et al., 2009; Dunkley et al., (2012); Luyten et al. (2007). A Dependência e Auto-criticismo correlacionam-se significativamente com outras medidas de funcionamento psicológico e psicopatologia. $\mathrm{O}$ factor de Auto-criticismo correlaciona-se com medidas de sintomas depressivos, como o Beck Depression Inventory. $\mathrm{O}$ factor de Dependência apresenta correlações mais baixas, por vezes não significativas com 
essas medidas de sintomas depressivos. A Dependência e Auto-criticismo são factores independentes entre si (veja-se Blatt, 2004; vejase também Blatt, 1995; Blatt et al., 1982; Campos, Besser, \& Blatt; 2013; Fazaa \& Page, 2003).

Investigação subsequente (Blatt, Zohar, Quinlan, Zuroff, \& Mongrain, 1995; Blatt, Zohar, Quinlan, Luthar, \& Hart, 1996; Rude \& Burnham, 1995) mostrou que se podem considerar dois subfactores no factor de Dependência - um sub-factor menos adaptativo, Necessidade; e um sub-factor mais adaptativo, Contacto (ou Relacionamento). De acordo com estudos anteriores, a Necessidade mas não o Contacto relaciona-se e prevê a ocorrência de sintomas depressivos (e.g., Campos et al., 2013).

Embora o QED tenha sido inicialmente desenvolvido como um instrumento de auto-relato para avaliar dois tipos de experiencias depressivas anacliticas/dependentes e introjectivas/de autocríticismo, estudos posteriores, quer com amostras clínicas, quer com amostras não clínicas, demonstraram a capacidade do instrumento para avaliar mais genericamente as características anaclíticas e introjectivas da personalidade ou, dito de outra forma, para avaliar as dimensões do relacionamento e da auto-definição de uma forma mais abrangente (Blatt, 2008). Na investigação empírica, a dependência e o auto-criticismo têm sido considerados traços ou características associadas às duas configurações psicopatológicas, ou estilos de personalidade que podem predispor os sujeitos não só à depressão, mas a outras formas de mal-estar ou distress (e.g., Bers, Besser, Harpaz- Rotem, \& Blatt, 2013; Besser \& Priel, 2003; Besser, Vliegen, Luyten, \& Blatt, 2008; Blatt, 2004; Campos, Besser, \& Blatt, 2010, 2012, 2013).

Apesar da aceitação do QED, alguns investigadores têm apresentado algumas críticas ao instrumento, inclusivamente questionado a sua validade (e.g., Coyne \& Whiffen, 1995; Coyne, Thompson, \& Whiffen 2004; Desmet, Vanheule, \& Verhaeghe, 2006; Lehman et al., 1997; Viglione, Philp, Clemmey, \& Camenzuli, 1990). Uma das críticas que foi realizada tem a ver com o próprio sistema de cotação, demasiado complexo, e baseado em pesos factoriais, em que cada item entra na cotação dos diversos factores. Este tipo de sistema de cotação apresenta alguns problemas, nomeadamente o de promover correlações entre as diferentes escalas / factores, dado que uma parte da variância partilhada é variância espúria. Alguns autores (e.g., Desmet et al. 2007) questionam também o facto de se usar um sistema de cotação baseado numa análise factorial obtida com uma população universitária, quando o teste é utilizado com outro tipo de populações. Para evitar, entre outros problemas, o da cotação com base em pesos factoriais, foram construídas versões reduzidas do QED, algumas com um sistema de cotação unitário, mas Zuroff, Mongrain e Santor (2004), com base numa extensa revisão da literatura, recomendam o uso do QED original (forma longa) sempre que possível.

Zuroff et al. (2004) concluem, também, que os conceitos propostos por Blatt são dimensões contínuas que podem ser identificadas e medidas de forma independente relativamente a outros constructos, como o neuroticíssimo e a depressão. Diversos esforços foram realizados no sentido de traduzir a adaptar o QED para outras línguas, incluindo o português, o francês, o alemão e o sueco.

Num estudo prévio com a versão portuguesa (Campos et al., 2013), são apresentados os resultados obtidos num programa de validação. Os autores estudaram a equivalência linguística da medida, a consistência interna, a precisão testereteste, a estrutura factorial e a validade convergente, discriminante, de critério e preditiva, usando diversas amostras não clínicas e uma amostra clínica. Verificou-se, por exemplo, que relativamente à estrutura factorial obtida com uma amostra de 488 estudantes universitários, os itens com saturações superiores a .40 nos três factores eram muito similares aos itens referidos por Blatt et al, (1979). No entanto, a ordem com que os factores emergiram na solução factorial da versão portuguesa era ligeiramente diferente da solução factorial da versão americana. Na solução factorial da versão portuguesa, o factor de auto-criticismo emergiu como primeiro factor, a dependência como segundo e a eficácia como terceiro factor. Verificou-se também que a congruência entre a solução factorial obtida com a amostra de 448 universitários e a solução factorial obtida com uma amostra de 405 indivíduos da comunidade era muito elevada (coeficiente de congruência de .92). Os resultados foram globalmente positivos e 
mostraram que a versão portuguesa do QED apresentava características psicométricas aceitáveis.

No entanto, algumas limitações identificadas pelos autores no programa de validação, nomeadamente um reduzido número de participantes na amostra utilizada para avaliar a precisão teste-reteste, mostram a necessidade de estudos psicométricos adicionais. Uma outra limitação incidiu nas correlações entre todos os factores do QED, que não foram apresentadas, uma vez que os autores se focaram exclusivamente nas correlações entre a Dependência e o Auto-criticismo. A amostra da comunidade que participou no programa de validação era composta apenas por 405 indivíduos e, provavelmente, não foi suficientemente grande para desenvolver uma base de dados que permita a comparação dos resultados portugueses com os resultados obtidos com amostras de outros países, em estudos interculturais.

O objectivo do presente estudo é apresentar dados psicométricos adicionais da versão portuguesa do Questionário de Experiências Depressivas com uma amostra maior de adultos da comunidade. Esta amostra de 1545 indivíduos contém os 405 indivíduos que participaram no programa de validação da versão portuguesa do QED (Campos et al., 2013). Apresentaremos resultados descritivos para os factores do QED e testaremos também a consistência interna e a validade convergente do instrumento, utilizando uma medida adicional, a Center for the Epidemiologic Studies of Depression Scale (CESD; Radloff, 1977), dado que, como foi dito anteriormente, as dimensões de personalidade propostas por Blatt (2004; Blatt \& Zuroff, 1992) e avaliadas pelo QED podem predispor os indivíduos à depressão. Também iremos comparar os indivíduos do género masculino e do género feminino relativamente aos valores médios obtidos nos factores do QED. Dado que Campos et al. (2013) avaliaram a estabilidade temporal do QED com uma amostra reduzida ( $n=63)$, no presente estudo testaremos a estabilidade temporal dos factores do QED numa amostra maior de jovens adultos $(\mathrm{N}=165)$ num período de cinco meses.

\section{Metodologia}

\section{Amostras, medidas e procedimentos}

A primeira amostra foi uma amostra extensa da comunidade composta por 1545 indivíduos adultos, que se voluntariaram para participar. Setecentos e cinquenta participantes eram do género masculino e 795 do género feminino As idades variaram entre os 19 e os 69 anos $(M=36.91, \quad D P=12.26)$. A maioria dos participantes era caucasiana e o nível educacional variou entre os 4 e os $21 \operatorname{anos}(M=12.02$, $D P=3.25)$. Todos os protocolos foram recolhidos em sessões individuais por assistentes de investigação previamente treinados. Os participantes não foram pagos ou compensados de outra forma pela sua participação e as instruções foram apresentadas em formato escrito. A investigação foi apresentada como respeitando à personalidade e a estados emocionais de indivíduos adultos. As guidelines da Ordem dos Psicólogos Portugueses foram seguidas e os participantes assinaram um termo de consentimento informado antes de responderem.

Para além do QED, e para avaliar sintomas depressivos, os participantes responderam à versão portuguesa (Gonçalves \& Fagulha, 2004) da Center for Epidemiologic Studies Depression Scale (CES-D; Radloff, 1977). A CES-D é um instrumento constituído por 20 itens a serem respondidos numa escala de Likert de 4 pontos, para a avaliar a frequência de sintomas depressivos na semana anterior. Os itens foram seleccionados de escalas de depressão já validadas, de modo a que os componentes mais importantes da sintomatologia depressiva ficassem contemplados. Os componentes em causa incluem o humor depressivo, sentimentos de culpa e desvalorização, sentimentos de desamparo e desespero, lentificação psicomotora, perda de apetite e perturbações do sono. A escala apresenta consistência interna e validade convergente adequadas. A versão portuguesa apresenta características psicométricas aceitáveis (veja-se Gonçalves \& Fagulha, 2004). O alfa de Cronbach em diferentes amostras variou entre .87 e 92 .

Uma segunda amostra de conveniência de 270 estudantes universitários foi recrutada numa universidade portuguesa e foi avaliada em dois 
momentos diferentes. O segundo momento de recolha de dados ocorreu em média cinco meses depois ( $M=20$ semanas, $D P=4.4$ semanas). Vinte e cinco estudantes não participaram no Momento 2 e 40 foram excluídos porque deram uma identificação incorrecta, não permitindo emparelhar os protocolos do Momento 1 e do Momento 2. Adicionalmente 40 participantes foram também excluídos devido a um número elevado de itens omissos ou devido a protocolos inválidos. Assim, uma amostra final de 165 participantes, 124 indivíduos do género feminino (75.2\%) e 41 indivíduos do género masculino (24.8\%), com uma idade que variou entre os $18 \mathrm{e}$ os 45 anos $(M=20.2, \quad D P=3.2 ; \quad$ Mediana=20) participou neste estudo. Apenas $3.6 \%$ dos participantes tinha 26 ou mais anos de idade. Quanto ao ano do curso frequentado, 30.3\% dos estudantes estava no primeiro ano do seu curso, $31.5 \%$ estava no segundo ano, $16.4 \%$ estava no terceiro ano, $10.9 \%$ no quarto ano, $3.0 \%$ no quinto ano e $7.9 \%$ não forneceu esta informação.

Os participantes voluntariaram-se e assinaram um termo de consentimento informado. Em pequenos grupos de cerca de 20 estudantes, em sala de aula, os participantes responderam à versão portuguesa do QED. As instruções foram apresentadas por escrito. Os protocolos foram recolhidos por uma assistente de investigação. Dado que este estudo tinha dois momentos de recolha de dados, pedimos aos participantes que escrevessem as iniciais do seu nome completo de modo a poder emparelhar os protocolos de ambos os momentos, mantendo ainda assim o anonimato de resposta. Os indivíduos não foram compensados monetariamente pela sua participação.

\section{Procedimentos de análise estatística}

Apresentamos resultados descritivos para os factores do QED e comparamos os resultados médios de ambos os géneros usando testes $\mathrm{t}$ para amostras independentes. Os valores das correlações entre os factores do QED foram obtidos através da correlação de Pearson. Esta mesma correlação de Pearson foi utilizada para calcular os valores de correlação entre os factores do QED e a CES-D, no sentido de testar a validade convergente do instrumento. A precisão teste-reteste foi avaliada calculando as correlações de Pearson entre os resultados obtidos nos factores do QED em dois momentos diferentes, com num intervalo de cinco meses.

\section{Resultados}

Os valores das médias e desvios-padrão nos cinco factores do QED obtidos pelos indivíduos da amostra da comunidade são apresentados no quadro 1. As comparações entre os indivíduos do género masculino e do género feminino da amostra da comunidade em Dependência, Autocriticismo, Eficácia, Necessidade e Contacto, indicaram que a Dependência foi significativamente mais elevada nas mulheres, $t(1543)=10.04, p<.001$ ( $\mathrm{d}$ de Cohen=.51), mas não se encontraram diferenças significativas no Autocriticismo e na Eficácia. A Necessidade foi significativamente mais elevada nas mulheres $t(1543)=7.13, p<.001$ (d de Cohen=.36), bem como o Contacto $t(1543)=6.02, p<.001(\mathrm{~d} d e$ Cohen=.31).

Quadro 1. Médias e desvios-padrão nos factores do QED

\begin{tabular}{lccc}
\hline Factor & Género & $\mathrm{M}$ & $\mathrm{DP}$ \\
\hline Dependência & Homens & -.67 & .83 \\
& Mulheres & -.24 & .85 \\
Auto-criticismo & Homens & -.51 & .99 \\
& Mulheres & -.53 & .99 \\
Eficácia & Homens & -.38 & 1.17 \\
& Mulheres & -.48 & 1.11 \\
Necessidade & Homens & -.26 & .78 \\
& Mulheres & .03 & .84 \\
Contacto & Homens & -.43 & .94 \\
& Mulheres & -.14 & .97 \\
\hline Nota: $N j=1545 ;$ Homens, $N=750 ;$ Mulheres, $N=795$
\end{tabular}

$\mathrm{Na}$ amostra total da comunidade, os alfas de Cronbach foram .73, .79, .74, .79 e .79, respectivamente, para os factores de Dependência, Auto-criticismo, Eficácia, Necessidade e Contacto. Na amostra de jovens adultos, os alfas de Cronbach foram .77, .78, .68, .84 e .84 para Dependência, Auto-criticismo, Eficácia, Necessidade e Contacto, respectivamente. Nesta mesma amostra de jovens adultos, a Dependência avaliada no Momento 1 correlacionou-se positiva e significativamente com a Dependência avaliada no Momento 2 ( $r=.75, p<.001)$. O Auto-criticismo avaliado no Momento 1 também se correlacionou 
positiva e significativamente com o Autocriticismo avaliado no Momento 2 ( $r=.77$, $p<.001)$, bem como a Eficácia $(r=.62, p<.001)$. A Necessidade no Momento 1 também se correlacionou positiva e significativamente com a Necessidade no Momento 2 ( $r=.79, p<.001)$, bem como o Contacto no Momento 1 e no Momento 2 ( $r=.64, p<.001)$.

O Quadro 2 apresenta as correlações entre os factores do QED e a versão portuguesa da CES-D na amostra da comunidade. A Dependência correlacionou-se significativamente com a Eficácia $(r=.17, p<.001)$, com a Necessidade $(r=.62, p<.001)$ e com o Contacto $(r=.77$, $p<.001)$. O Auto-criticismo correlacionou-se com a Eficácia $(r=.10, p<.001)$, com a Necessidade $(r=.41, p<.001)$ e com o Contacto $(r=.14, p<.001)$.

A Eficácia correlacionou-se com a Necessidade $(r=-.34, \quad p<.001)$ e com o Contacto $(r=.58$, $p<.001)$. A Necessidade correlacionou-se com o Contacto $(r=.15, p<.001)$.

$\mathrm{Na}$ amostra da comunidade a Dependência correlacionou-se significativamente com a versão portuguesa da CES-D $(r=.20, p<.001)$, bem como com o Auto-criticismo $(r=.53, p<.001)$ e a Necessidade $(r=.44, p<.001)$. O Contacto $(r=.12$, $p<.001)$ e a Eficácia $(r=-.13, p<.001)$ também se correlacionaram significativamente com a versão Portuguesa da CES-D, mas ambos os valores apresentam magnitudes do efeito reduzidas.

Quadro 2. Correlações entre os factores do QED e a CES-D

\begin{tabular}{lccccc}
\hline Variáveis & 1 & 2 & 3 & 4 & 5 \\
\hline 1. Dependência & ---- & & & & \\
2. Auto-criticismo & .01 & & & & \\
3. Eficácia & $.17^{*}$ & $.10^{*}$ & & & \\
4. Necessidade & $.62^{* \mathrm{a}}$ & $.41^{*}$ & $-.34^{*}$ & & \\
5. Contacto & $.77^{* \mathrm{a}}$ & $.14^{*}$ & $.58^{*}$ & $.15^{*}$ & \\
6. CES-D & $.20^{*}$ & $.53^{*}$ & $-.13^{*}$ & $.44^{*}$ & $.12^{*}$ \\
\hline
\end{tabular}

Nota: * $p<.001{ }^{a}$ Estas correlações são contaminadas, pelo facto de serem correlações parte-todo.

\section{Discussão}

O estudo visou apresentar dados psicométricos adicionais para a versão portuguesa do QED. Os resultados apoiam a robustez psicométrica da versão portuguesa do inventário, complementando os resultados obtidos previamente noutro estudo. Os diferentes factores da versão portuguesa do QED apresentaram valores aceitáveis de consistência interna e de estabilidade temporal, confirmando que o QED avalia características estáveis da personalidade. A estabilidade temporal num período de cinco meses parece sublinhar também a robustez da avaliação da experiência fenomenológica da depressão como forma de estudo da personalidade normal e depressiva.

Consistentemente com resultados anteriores (e.g., Aube \& Whiffen, 1996; Zuroff et al., 1990), as mulheres da amostra da comunidade apresentaram resultados significativamente mais elevados do que os homens em Dependência. Também consistentemente com resultados anteriores, os homens da amostra da comunidade pontuaram mais alto, mas não significativamente mais alto do que as mulheres em Auto-criticismo (e.g., Aube \& Whiffen, 1996; Zuroff \& Fitzpatrick, 1995). Este resultado, contudo, é contrário ao de outros estudos onde se verificou que a diferença de géneros no factor de Autocriticismo era significativa (Dunkley et al., 1997; Zuroff et al., 1990). Poucos estudos anteriores apresentaram os valores médios nos factores de Necessidade e Contacto, separadamente, por géneros. Consistentemente com alguns resultados anteriores (Bacchiochi, Bagby, Cristi, \& Watson, 2003), as mulheres da nossa amostra da comunidade pontuaram significativamente mais alto do que os homens, quer em Necessidade, quer em Contacto. No entanto, outros estudos (Rude \& Burnham, 1995) encontraram diferenças significativas para Contacto, mas não para Necessidade.

A ausência de uma correlação significativa entre Dependência e Auto-criticismo é consistente com a literatura, com a maioria dos estudos a não obterem correlações significativas entre estes factores do QED em amostras não clínicas (vejase Blatt, 2004). Contudo, o Auto-criticismo correlacionou-se significativamente com a Necessidade, o componente desadaptativo da Dependência e, marginalmente, com o Contacto. O Contacto, no entanto, correlacionou-se positiva e fortemente com a Eficácia, o que confirma que esta componente mais madura da Dependência é uma dimensão adaptativa. A Necessidade correlacionou-se marginalmente com o Contacto. Em estudos anteriores, estas duas dimensões 
correlacionaram-se uma com a outra numa amostra de estudantes, mas não numa amostra clínica (McBride, Zuroff, Bacchlochi \& Bagby, 2006).

O factor de Auto-criticismo correlacionou-se significativamente com a CES-D. Este resultado é consistente com resultados obtidos com outras medidas de sintomas depressivos, quer em amostras clínicas, quer em amostras não clínicas (e.g., Blatt, 2004; Santor et al., 1997). A correlação entre a CES-D e o factor de Dependência foi mais baixa. As correlações da Dependência com medidas de sintomas depressivos são, em média, mais baixas (e muitas vezes não significativas) especialmente em amostras não clínicas (e.g., Blatt, 2004; Dunkley et al., 1997). O resultado agora obtido confirma a importância de considerar os sub-factores do factor de Dependência do QED. De acordo com os nossos resultados, o Contacto correlacionou-se apenas marginalmente com a CES-D. Esta diferente associação com medidas de sintomas depressivos é consistente com resultados obtidos previamente relativos a estes dois sub-factores (Besser \& Priel, 2005, 2008; Blatt et al., 1995; Campos et al., 2010). Diversos estudos indicam que o factor de Dependência contém, quer uma componente de vulnerabilidade, quer uma componente de resiliência (e.g., Blatt et al., 1995; Blatt et al., 1996; Campos et al., 2011; Priel \& Besser, 1999; Rude \& Burnham, 1993), indicando também a importância de diferenciar um subfactor desadaptativo e um sub-factor adaptativo, contidos no factor de Dependência (e.g., Campos, Besser, Ferreira, \& Blatt, 2012).

Relativamente às correlações entre Contacto e Necessidade com a CES-D, realizámos uma análise complementar em separado para homens e mulheres. Enquanto a Necessidade se correlacionou significativamente com os sintomas depressivos avaliados pela CES-D, quer nos homens $(r=.39, \quad p<.001)$, quer nas mulheres ( $r=.45, p<.001)$, o Contacto só se correlacionou significativamente com os sintomas depressivos nas mulheres $(r=.18, p<.001)$. Poderá acontecer que a Necessidade seja desadaptativa, quer para os homens, quer para as mulheres, porque promove uma activação da ruminação. A diferença de géneros na vulnerabilidade para a depressão pode vir do Contacto (McBride \& Bagby, 2006). De acordo com McBride e Bagby "a interacção entre a ruminação e a dependência interpessoal pode contribuir para a diferença de géneros na depressão. A Necessidade Interpessoal [parece resultar] em depressão, quer nos homens, quer nas mulheres, através do papel mediador da ruminação. Para as mulheres, o Contacto interpessoal é uma dimensão adaptativa e saudável do crescimento e do bem-estar. No entanto, sendo mais focadas nos relacionamentos, as mulheres são mais vulneráveis a stressores interpessoais, que trazem consequentemente o risco de activar a ruminação, e em última instância a depressão" (p. 189, 191).

A Eficácia parece ser, de acordo com os nossos resultados, um factor protector ou de resiliência (Bers, Harpaz-Rotem, Besser, \& Blatt, 2013). Correlacionou-se negativamente com os sintomas depressivos, embora o valor de correlação tenha apresentado uma magnitude do efeito reduzida. Correlacionou-se positiva e fortemente com o Contacto. Campos, Besser e Blatt (2012), por exemplo, verificaram que a Eficácia pode ser um factor protector contra a ideação e comportamentos suicidas. No presente estudo, a Eficácia correlacionou-se significativamente com o Auto-criticismo, apesar de uma magnitude do efeito reduzida do valor da correlação. De acordo com Blatt (2008), a Eficácia pode ser uma dimensão mais evoluída/madura da linha do desenvolvimento da auto-definição, assim como o Contacto é uma dimensão mais evoluída da linha do desenvolvimento do relacionamento interpessoal.

\section{Limitações e conclusões}

O presente estudo tem algumas limitações. Uma limitação importante é que a amostra da comunidade, embora extensa, não é representativa da população portuguesa adulta. Outra limitação é a de que neste estudo não se avaliou a validade discriminante.

No entanto, e de um modo geral, os dados obtidos no presente estudo indicam características psicométricas aceitáveis da versão portuguesa do QED e expandem e complementam os resultados obtidos previamente por Campos et al (2013). Os resultados são também consistentes com os obtidos com outras adaptações do QED. Sugerem que a Necessidade e o Auto-criticismo são 
factores estáveis da personalidade e que se relacionam com os sintomas depressivos. Os resultados também sugerem que a Eficácia e o Contacto podem ser factores protectores ou de resiliência e que a Dependência é um factor híbrido composto por duas componentes, uma mais evoluída e outra mais imatura: Contacto e Necessidade, respectivamente. Contudo, nas mulheres, e de acordo com os resultados, o Contacto pode conferir alguma vulnerabilidade aos sintomas depressivos. Os dois sub-factores, mas não o factor de Dependência, devem ser utilizados como medidas de traços da personalidade anaclítica na avaliação clínica e na investigação.

A adaptação e validação de instrumentos sólidos do ponto de vista psicométrico é sempre uma tarefa de investigação importante, especialmente se essas versões adaptadas puderem ser usadas na prática clínica e puderem também contribuir para o desenvolvimento e "afinação" de modelos teóricos (Casullo, 2009). Apesar das suas limitações, esperamos que a versão portuguesa do QED possa ser útil para clínicos e investigadores, nomeadamente para aqueles que adoptam uma abordagem psicodinâmica na avaliação da personalidade, da depressão e do mal-estar psicológico (Campos, aceite) e consideram o relacionamento e a auto-definição (Blatt, 2004, 2008) como duas dimensões globais ("higher order"), no funcionamento psicológico normal e no funcionamento psicológico desadaptativo (Luytem \& Blatt, 2016).

\section{Referências}

Aube, J., \& Whiffen, V. (1996). Depressive styles and social acuity: Further evidence for distinct interpersonal correlates of dependency and self-criticism. Communication Research, 23, 407-424. doi:10.1177/009365096023004004

Bacchiochi, J. R., Bagby, R. M., Cristi, C., \& Watson, J. (2003). Validation of connectedness and neediness as dimensions of dependency construct. Cognitive Therapy and Research, 27, 233-242.

Bers, S. A., Besser, A., Harpaz-Rotem, I., \& Blatt, S. J. (2013). Representations of self, mother and father: An empirical exploration of the dynamics of anorexia nervosa. Psychoanalytic Psychology, 32, 188209. doi:10.1037/a0032512

Besser, A., \& Priel, B. (2003). A multisource approach to self-critical vulnerability to depression: The moderating role of attachment. Journal of Personality, 71, 515555. doi:10.1111/1467-6494.7104002

Besser, A., \& Priel, B. (2005). Interpersonal relatedness and self-definition in late adulthood depression: Personality predispositions and protective factors. Social Behavior and Personality, 33, 351-382. doi:10.2224/sbp.2005.33.4.351

Besser, A., \& Priel, B. (2008). Attachment, depression, and fear of death in older adults: The roles of neediness and perceived availability of social support. Personality and Individual Differences, 44, 1711-1725. doi:10.1016/j.paid.2008.01.016

Besser, A., Vliegen, N., Luyten, P., \& Blatt, S. J. (2008). Vulnerability to postpartum depression from a psychodynamic perspective: Systematic empirical base commentary on issues raised by Blum (2007). Psychoanalytic Psychology, 25, 392-410. doi:10.1037/0736-9735.25.2.392

Bibring, E. (1953). The mechanism of depression. In P. Greenacre (Ed.), Affective disorders (pp. 13-48). New York, NY: International University Press.

Blatt, S. (1974). Levels of object representation in anaclytic and introjective depression. Psychoanalytic Study of the Child, 29, 107157.

Blatt, S. J. (1995). The destructiveness of perfectionism: Implications for the treatment of self-depression. American Psychologist, 53, 103-120. doi:10.1037/0003-066X.50.12.1003

Blatt, S. J. (2004). Experiences of depression: Theoretical, research e clinical perspectives. Washington, DC: American Psychological Association.

Blatt, S. J. (2008). Polarities of experience: Relatedness e self-definition in personality development, psychopathology, e the therapeutic process. Washington, DC: American Psychological Association Press.

Blatt, S., D'Afflitti, J., \& Quinlan, D. (1976). Experiences of depression in normal young 
adults. Journal of Abnormal Psychology, 85, 383-389.

Blatt, S., D’Afflitti, J., \& Quinlan, D. (1979). Depressive Experiences Questionnaire. Unpublished Manual, Yale University, New Haven, CT.

Blatt, S., \& Homann, E. (1992). Parent-child interaction in the etiology of dependent and self-critical depression. Clinical Psychology Review, 12, 47-91. doi:10.1016/02727358(92)90091-L

Blatt, S. J., Quinlan, D. M., Chevron, E. S., McDonald, C., \& Zuroff, D. (1982). Dependency and self-criticism: Psychological dimensions of depression. Journal of Consulting and Clinical Psychology, 50, 113124. doi:10.1037/0022-006X.50.1.113

Blatt, S. J., Zohar, A. H. Quinlan, D. M., Zuroff, D. C., \& Mongrain, M. (1995). Subscales within the dependency factor of the Depressive Experiences Questionnaire. Journal of Personality Assessment, 64, 319339. doi:10.1207/s15327752jpa6402_11

Blatt, S., Zohar, A., Quinlan, D., Luthar, S., \& Hart, B. (1996). Levels of relatedness within the dependêncy factor of the Depressive Experiences Questionnaire for Adolescents. Journal of Personality Assessment, 67, 52-71. doi:10.1207/s15327752jpa6701_4

Blatt, S. J., \& Zuroff, D. C. (1992). Interpersonal relatedness and self-definition: Two prototypes for depression. Clinical Psychology Review, 12, 527-562. doi:10.1016/0272-7358(92)90070-O

Campos, R. C. (2017). Do processo de avaliação da personalidade em contextos clínicos ao diagnóstico psicodinâmico: Contributos para uma avaliação psicológica psicodinâmica. Revista Iberoamericana de Diagnóstico y Evaluación - e Avaliação Psicológica, 4,4456.

Campos, R. C., Besser, A., \& Blatt, S. J. (2010). The mediating role of self-criticism and dependency in the association between perceptions of maternal caring and depressive symptoms. Depression and Anxiety, 27, 11491157. doi:10.1002/da.20763

Campos, R. C., Besser, A., \& Blatt, S. J. (2011). The relationships between defenses and experiences of depression: An exploratory study. Psychoanalytic Psychology, 28, 196208. doi:10.1037/a0022420

Campos, R. C., Besser, A., \& Blatt, S. J. (2012). Distress mediates the association between personality predispositions and suicidality: A preliminary study in a Portuguese community sample. Archives of Suicide Research, 16, 4458. doi:10.1080/13811118.2012.640583

Campos, R. C., Besser, A., Ferreira, R., \& Blatt, S. J. (2012). Self-criticism, neediness, and distress among women undergo breast cancer treatment: A Preliminary test of the moderating role of adjustment to illness. International Journal of Stress Management, 19, 151-174.

Campos, R. C., Besser, A., \& Blatt, S. J. (2013). The Portuguesa version of the Depressive Experiences Questionnaire: Results from a validation program in clinical and non clinical samples. Spanish Journal of Psychology, 16, 1-13. doi:10.1017/sjp.2013.104

Casullo (2009). La evaluacion psicolgoica: Modelos, técnicas e contextos. Revista Iberoamericana de Diagnóstico y Evaluación - e Avaliação Psicológica, 27, 9-28.

Cohen, M. B., Baker, G., Cohen, R. A., FromReichman, F., \& Weigert, E. V. (1954). An intensive study of twelve cases of manicdepressive psychosis. Psychiatry, 17, 103137.

Coyne, J. C., \& Whiffen, V. E. (1995). Issues in personality as diathesis for depression: The case of sociotropy-dependêncy and autonomyself-criticism. Psychological Bulletin, 118, 358-378. doi:10.1037/0033-2909.118.3.358

Coyne, J. C., Thompson, R., \& Whiffen, V. (2004). Is the Promissory note of personality as vulnerability to depression in default? Reply to Zuroff, Mongrain, e Santor (2004). Psychological Bulletin, 130, 512-517. doi:10.1037/0033-2909.130.3.512

Desmet, M., Vanheule S., Groenvynck, H., Verhaeghe, P., Vogel, J., \& Bogaerts, S. (2007). The Depressive Experiences Questionnaire: An inquiry to the different scoring procedures. European Journal of Psychological Assessment, 23, 89-98. doi:10.1027/1015-5759.23.2.89

Desmet, M., Vanheule, S., Meganck, R., Verhaeghe, P., \& Bogaerts, S. (2007). The 
Depressive Experiences Questionnaire: On using a student-based scoring program for a clinical sample. Psychological Reports, 101, 587-596. doi:10.2466/PR0.101.6.587-596

Desmet, M., Vanheule, S., \& Verhaeghe, P (2006). Dependency, self-criticism, and the symptom specificity hypothesis in a depressed clinical sample. Social Behavior and Personality, 34, 1017-1026. doi:10.2224/sbp.2006.34.8.1017

Desmet, M., Verhaeghe, P., Van Hoorde, H., Meganck, R., Vanheule, S., \& Murphy, C. (2009). The Depressive Experiences Questionnaire as a measure of psychoanalytic constructs reported to be measured. Psychological Reports, 105, 714-720.

Dunkley, D. M., Blankstein, K. R., \& Flett, G. L. (1997). Specific cognitive-personality vulnerability styles in depression and the fivefactor model of personality. Personality and Individual Differences, 23, 1041-1053. doi:10.1016/S0191-8869(97)00079-2

Dunkley, D. M., Schwartzman, D., Looper, K. J., Sigal, J. J., Pierre, A., \& Kotowycz, M. A. (2012). Perfectionism dimensions and dependency in relation to personality vulnerability and psychosocial adjustment in patients with coronary artery disease. Journal of Clinical Psychology in Medical Settings, 19, 211-223. doi:10.1007/s10880-011-9271-2

Fazaa, N., \& Page, S. (2003). Dependency and self-criticism as predictors of suicidal behavior. Suicide e Life-Threatening Behavior, 33, 172-185. DOI:10.1521/suli.33.2.172.22777

Freud, S. (1917/1980). Luto e melancolia (Edição standard brasileira das obras psicológicas completas de Sigmund Freud -vol. XIV). Rio de Janeiro, Brasil: Imago Editora.

Gonçalves, B., \& Fagulha, T. (2004). The Portuguese version of the Center for Epidemiologic Studies Depression Scale (CES-D). European Journal of Psychological Assessment, 20, 339-348.

Lehman, A. K., Ellis, B., Becker, J., Rosenfarb, I., Devine, R., Khan, A., \& Reichler, R. (1997). Personality and depression: A validation study of the Depressive Experiences Questionnaire. Journal of Personality Assessment, 68, 197210. doi:10.1207/s15327752jpa6801_16
Luyten, P., \& Blatt, S. J. (2016). A Hierarchical multiple-level approach to the assessment of interpersonal relatedness and self-definition: Implications for research, clinical practice, and DSM planning. Journal of Personality Assessment, 98, 5-13. doi:10.1080/00223891. 2015.1091773

Luyten, P., Sabbe, B., Blatt, S., Meganck, S., Jansen, B., De Grave, C., (...) \& Corveleyn, J. (2007). Dependency and self-critricism: Relationship with major depressive disorder, severity of depression, and clinical presentation. Depression and Anxiety, 24, 586-596. doi:10.1002/da.20272

McBride, C., \& Bagby, R. M. (2006). Rumination and interpersonal dependency: Explaining women's vulnerability to depression. Canadian Psychology, 47, 184-194. doi:10.1037/cp2006008

McBride, C., Zuroff, D., Bacchlochi, J., \& Bagby, R. M. (2006). Depressive Experiences Questionnaire: Does it measure maladaptive and adaptive forms of Dependency? Social Behavior and Personality, 34, 1-16. doi:10.2224/sbp.2006.34.1.1

Priel, B., \& Besser, A. (1999). Vulnerability to postpartum depressive symptomatology: Dependency, self-criticism and the moderating role of antenatal attachment. Journal of Social and Clinical Psychology, 18, 240-253. doi:10.1521/jscp.1999.18.2.24

0

Quimette, P. C., \& Klein, D. N. (1993). Convergence of psychoanalytic and cognitivebehavioral theories of depression: An empirical review and new data on Blatt's and Beck's models. In J. M. Masling, \& R. F. Bornstein (Eds.), Psychoanalytic Perspectives on Psychopathology. Washington, DC: American Psychological Association.

Radloff, L. S. (1977). The CES-D Scale: A selfreport depression scale for research in the general population. Applied Psychological Measurement, 1, 385-401.

Rude, S. S., \& Burnham, B. L. (1993). Do interpersonal and achievement vulnerabilities interact with congruent events to predict depression? Comparison of DEQ, SAS, DAS, and combined scales. Cognitive Therapy \& Research, 17, 531-548. doi:10.1007/BF01176 


\section{7}

Rude, S. S., \& Burnham, B. L. (1995). Connectedness and neediness: Factors of the DEQ and SAS dependency scales. Cognitive Therapy \& Research, 19, 323-340. doi:10.1007/BF02230403

Santor, D. A., Zuroff, D. C., \& Fielding, A. (1997). Analysis and revision of the Depressive Experiences Questionnaire: Examining scale performance as a function of scale length. Journal of Personality Assessment, 69, 145-163. doi:10.1207/s15327 752jpa6901_8

Zuroff, D. C., Mongrain, M., \& Santor, D. A. (2004). Conceptualizing e measuring personality vulnerability to depression: Comment on Coyne and Whiffen (1995). Psychological Bulletin, 130, 489-511. doi:10.1037/0033-2909.130.3.489

Zuroff, D. C., Quinlan, D. M., \& Blatt, S. J. (1990). Psychometric properties of the Depressive Experiences Questionnaire in a college population. Journal of Personality Assessment, $\quad 55, \quad 65-72$. doi:10.1080/00223891.1990.9674047 